\title{
Effect of Cytokinin on Multiple Shoot Regeneration in Shoot Apical Culture of Physalis minima L. - An Important Fruit and Medicinal Plant
}

\author{
Kumari Anjani* and Harsh Kumar \\ Department of Agricultural Biotechnology and Molecular Biology, Dr. Rajendra Prasad \\ Central Agricultural University, Pusa-848125, Bihar, India \\ *Corresponding author
}

\section{A B S T R A C T}

\begin{tabular}{|l|}
\hline Ke y w or d s \\
Cytokinin, \\
Micropropagation, \\
BAP, TDZ, KIN, \\
Multiple shoot \\
\hline Article Info \\
\hline Accepted: \\
26 March 2018 \\
Available Online: \\
10 April 2018 \\
\hline \hline
\end{tabular}

Shoot apices of Physalis minima L. were cultured on fifteen full strength MS medium supplemented with various concentrations of cytokinins (BAP, KIN and TDZ). Tissue culture responses namely elongation of existing shoot and differentiation of multiple shoots were observed from the cultured explants. The establishment of cultured explants was not affected by the cytokinin concentration in the medium but overall shoot apices showed better establishment on medium supplemented with BAP than other cytokinins. Majority of established explants showed elongation of existing shoot followed by differentiation of multiple shoots. The maximum frequency of elongation of existing shoot $(86.13 \pm 0.94)$ was observed in MS 2 which has BAP at $1.0 \mathrm{mg} / \mathrm{L}$ concentration and minimum was observed on MS $10(\mathrm{KIN} 2.5 \mathrm{mg} / \mathrm{L})$. The frequency of multiple shoot differentiation was less than shoot elongation and medium with BAP $(1.0 \mathrm{mg} / \mathrm{l})$ showed the best response of $75.15 \pm 0.52$ percent. The multiple shoots developed directly from the shoot apices, without the formation of callus. Thus, the results of this investigation can be used to develop a very efficient micropropagation protocol for this economically important plant.

\section{Introduction}

India is the home of about 15000 species of flowering plants but only 800 species have been domesticated. The proportion of medicinal plants is still low. There are 3000 species of medicinal plants that have been officially documented but very few of them are grown on large scale and commercially exploited. Their limited availability and overexploitation is resulting in a gradual decline of such species. One such plant is Physalis minima L. In India, it grows like a weed throughout Andhra Pradesh, Bengal, Gujarat, Karnataka, Kerala, Maharashtra, Tamil Nadu and in many parts of Bihar (Usaizan et al., 2014 and Anjani and Kumar, 2018). Itbelongs to the family Solanaceae and is a small, delicate, annual herb having a height of 0.5-1.5m (Chothani and Vaghasiya, 2012; Ukwubile and Oise, 2016 and Anjani and Kumar, 2018). It has great medicinal value and a very good potential as a nonconventional fruit. The fruit is a good source of vitamin $\mathrm{C}$, protein, minerals, potassium, calcium, magnesium, iron and phosphorus. It 
has been widely used in Indian Traditional System of Medicines as diuretic, purgative, analgesic, anthelmintic, anti-inflammatory, antimicrobial, appetizer and bitter tonic (Sheeba et al., 2015). But the plant is considered a cosmopolitan weed and adequate measures have not been taken for its bulking and conservation. In vitro studies can be very useful in this context since rapid multiplication of various genotypes can be achieved through micropropagation. The tissue culture methods can also help in conservation of germplasm by providing large amount of propagating material in a short span of time with limited space requirement (Afroz et al., 2009).

The in vitro methods can provide large amount of propagative materials in a very short duration (Mungole et al., 2011). But the efficacy of micropropagation depends on the rapidity of multiple shoot formation. The shoot apex is found to be the most effective explant for multiple shoot formation in many plants (Solanki and Gupta, 2013). The multiple shoot formation in cultures is influenced by combination of plant growth regulators used for the culture besides other factors. The plant growth regulators particularly cytokinin play a very important role in the response of an explant to in vitro conditions. Thus, this article investigates the effect of cytokinin on multiple shoot regeneration in shoot apical culture of $P$. minima in an attempt to develop a highly efficient micropropagation protocol for this plant.

\section{Materials and Methods}

The $P$. minima plants grown in greenhouse were used as a source of explant for the present study. The shoot apices from 30 to 35 days old plants were used as explants. The explants were excised from plants, treated with five to six drops of Tween 20 and washed with running tap water for $30 \mathrm{~min}$ followed by rinsing with distilled water 4-5 times. These explants were then surface sterilized with $0.1 \%$ mercuric chloride for $4 \mathrm{~min}$ and rinsed with sterile distilled water 3-4 times.

The surface sterilized explants were treated with $70 \%$ ethanol for $30 \mathrm{sec}$ and thoroughly washed with sterile distilled water 3-5 times. The sterile explants were inoculated in culture tubes on Murashige and Skoog (MS) basal medium supplemented with various concentrations of cytokinin Benzyl amino purine (BAP), kinetin (KIN) and Thidiazuron (TDZ) (Table 1). Sixty tubes were inoculated on each medium in three replications. The tubes were incubated for $16 \mathrm{~h}$ photoperiod at $25 \pm 2^{\circ} \mathrm{C}$ under fluorescent light (3000 lux). The cultures were evaluated for establishment, elongation of existing shoot and differentiation of multiple shoots. The data were analyzed statistically according to completely randomized block design (CRD) and subjected to one way analysis of variance to test the significance of the observed result.

\section{Results and Discussion}

The shoot apices were inoculated on fifteen different MS medium having different combination of three cytokinins (BAP, KIN and TDZ) to establish shoot apical cultures. Three responses namely establishment, elongation of existing shoot and multiple shoot formation were recorded. The establishment of the cultures did not show any regular pattern and thus was not affected by the concentration and type of cytokinin used for the culture. However, the highest frequency of establishment $(96.11 \pm 0.56)$ was observed on MS 2 having BAP $1.0 \mathrm{mg} / \mathrm{L}$ and lowest $(75 \pm 0.96)$ on MS 10 having KIN $2.5 \mathrm{mg} / \mathrm{L}$. The highest average establishment was observed on the medium supplemented with BAP (88.78) and lowest on the medium containing KIN (82.89) (Table 2; Fig. 1). 
The cultures showed elongation of existing shoots 15-20 days after inoculation. All the medium showed this response but at different rates. While the maximum frequency of shoot elongation $(86.13 \pm 0.94)$ was observed on MS 2 (BAP $1.0 \mathrm{mg} / \mathrm{L})$, minimum was observed on MS 10 (KIN 2.5mg/L). The medium supplemented with TDZ had highest average percentage elongation of existing shoot (80.07) and lowest response was shown by the medium with KIN (63.33) (Table 2; Fig. 1). Nonetheless, the elongation of existing shoot was more intense on MS medium supplemented with BAP (Fig. 2).

The multiple shoots appeared after 20-25 days of inoculation. The frequency of multiple shoot formation was less than shoot elongation on all medium. The multiple shoot appeared on all the medium but MS 2 (BAP $1.0 \mathrm{mg} / \mathrm{L}$ ) was the best with highest frequency of differentiation of multiple shoot (75.15 \pm $0.52)$. The lowest response $(41.48 \pm 0.39)$ was observed on MS 10 (KIN $2.5 \mathrm{mg} / \mathrm{L}$ ). The highest average response was present on the medium with TDZ (69.23) and lowest on the medium with KIN (56.03) as is evident from Table 2 and Figure 1. But the medium containing BAP as supplement exhibited more number of multiple shoots per explant (Fig. 3). The multiple shoots formed on these medium were better and green and did not show vitrification. For all responses, MS 2 was found to be most effective media and MS 10 was found to be least effective. While the medium with TDZ showed best average number of most of the responses, the medium with BAP showed more extensive responses. The KIN was found to be the least effective cytokinin for all the responses. It was observed that as the concentration of hormones is increased, frequency of responses increase and then decline on further increasing the concentration. Thus, there is a best concentration for each cytokinin where the response is maximum and lower on either side
(Fig. 1). This concentration for each cytokinin varies with every response. For BAP, the most suitable concentration for all response is $1.0 \mathrm{mg} / \mathrm{L}$. For the medium containing KIN, while the best concentration for establishment and elongation of existing shoot is $1.5 \mathrm{mg} / 1$, for differentiation of multiple shoot is $0.5 \mathrm{mg} / \mathrm{L}$. For TDZ, the ideal concentration for all responses is $1.5 \mathrm{mg} / \mathrm{L}$ at which the responses are maximum.

In vitro techniques offer excellent opportunity for conservation of economically important plant species. Multiple shoot induction, proliferation and maintenance for longer period of time is governed by different culture conditions and plant growth regulators especially cytokinins (Intzaar et al., 2013). The present study was carried out in an attempt to identify the most suitable cytokinin and its most effective concentration for multiple shoot formation in shoot apical culture of $P$. minima plants.

Tissue culture responses namely establishment, elongation of existing shoot and differentiation of multiple shoots were observed from the cultured shoot apices. The frequency of establishment of culture did not show any particular pattern and thus, was not affected by the type and concentration of the cytokinin present in the medium. This can be explained on the fact that establishment of culture is defined only on the basis of presence or absence of infection and the healthy state of explants. The component of the media has no effect on these aspects and thus do not affect frequency. However, BAP proved to be a better cytokine in for establishment with highest average frequency of establishment (88.78). The lowest average frequency (82.89) was observed on the medium containing KIN. MS 2 was the most effective medium for establishment and MS 10 was the least effective. Similar results were obtained by Afroz et al., (2009) and Sheeba et al., (2015. 
Fig.1 Effect of type and concentration of cytokinin on shoot apical culture of Physalis minima L.

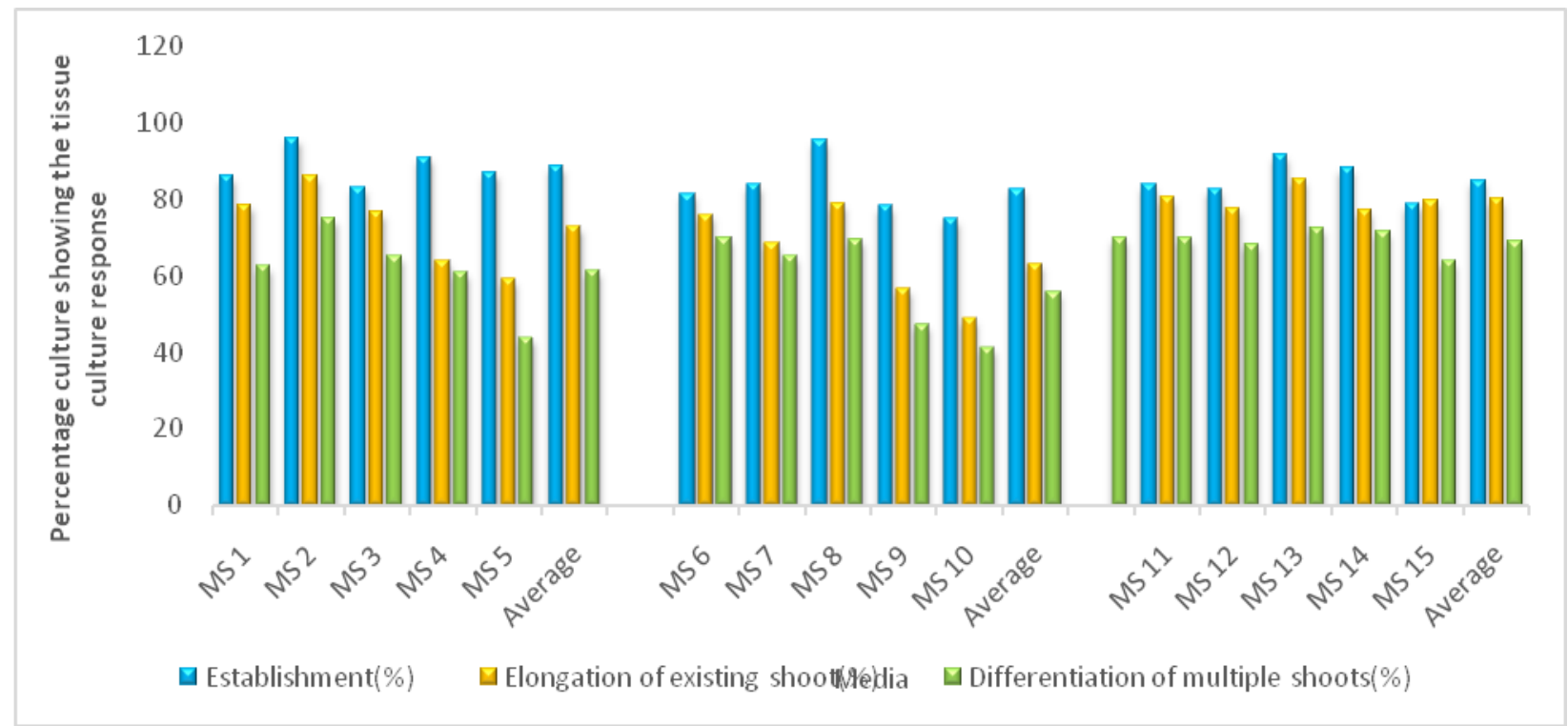

Fig.2 Elongation of existing shoot on different medium. A-MS 2 (MS + 1.0mg/L BAP); B- MS 7 $(\mathrm{MS}+1.0 \mathrm{mg} / \mathrm{L} \mathrm{KIN}) ; \mathrm{C}-\mathrm{MS} 12(\mathrm{MS}+1.0 \mathrm{mg} / \mathrm{L} \mathrm{TDZ})$
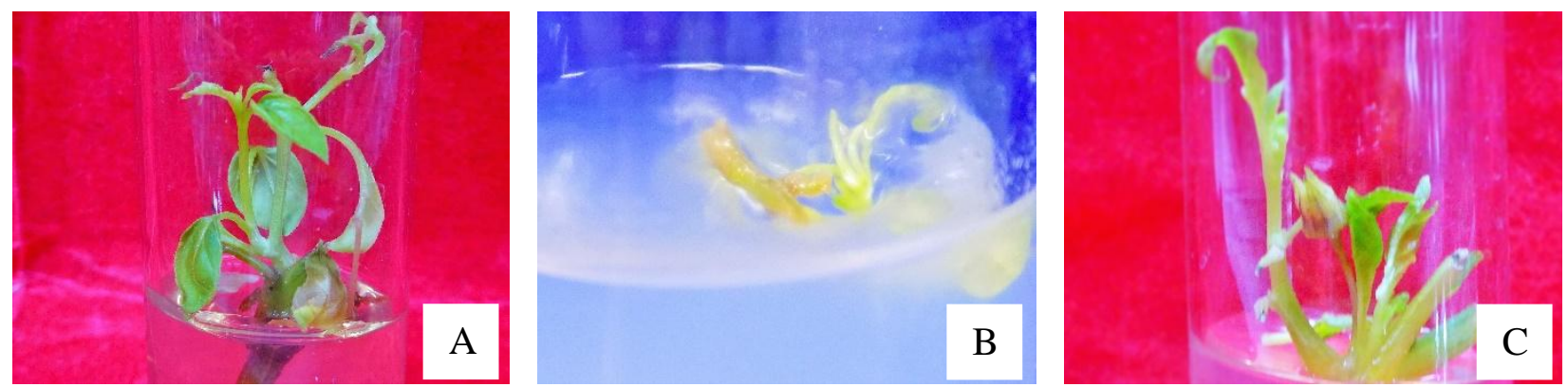

Fig.3 Differentiation of multiple shoot on different medium. A-MS 3 (MS + 1.5mg/L BAP); BMS 6 (MS + 0.5mg/L KIN); C- MS 13 (MS + 1.5mg/L TDZ)
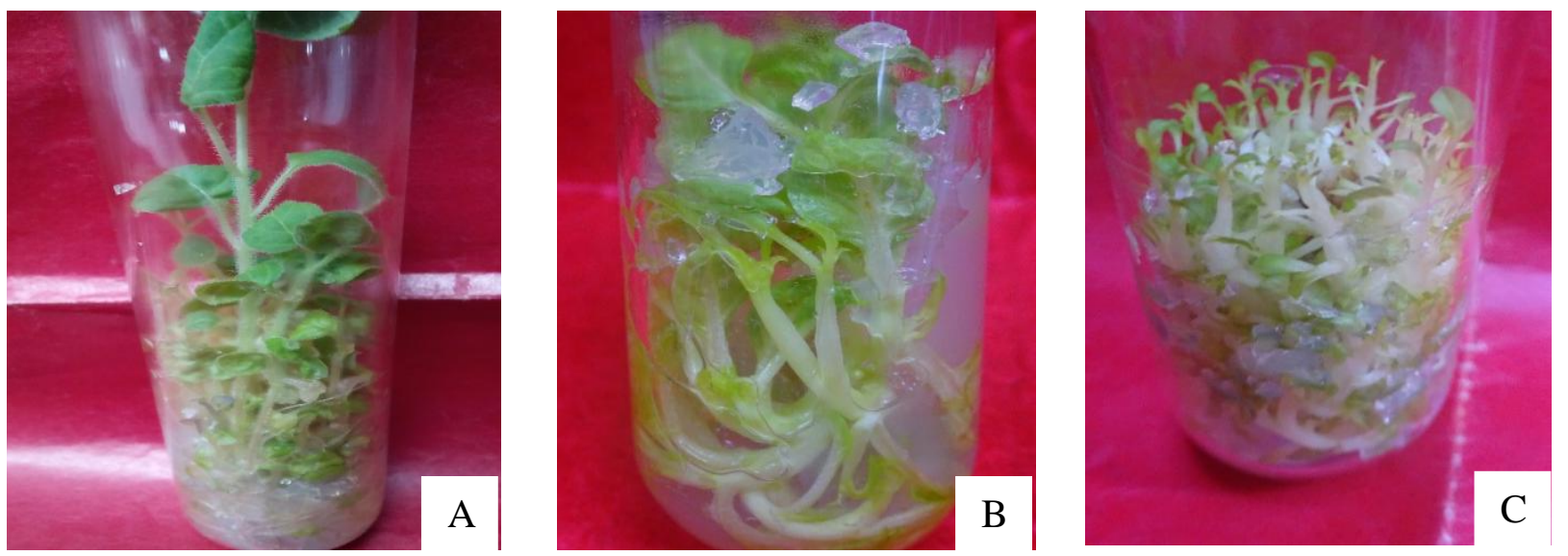
Table.1 The list of the medium used for the study

\begin{tabular}{|c|c|c|}
\hline Medium No. & Cytokinin Present & Concentration mg/L \\
\hline MS 1 & BAP & 0.5 \\
\hline MS 2 & BAP & 1 \\
\hline MS 3 & BAP & 1.5 \\
\hline MS 4 & BAP & 2.0 \\
\hline MS 5 & BAP & 2.5 \\
\hline MS 6 & KIN & 0.5 \\
\hline MS 7 & KIN & 1 \\
\hline MS 8 & KIN & 1.5 \\
\hline MS 9 & KIN & 2.0 \\
\hline MS 10 & KIN & 2.5 \\
\hline MS 11 & TDZ & 0.5 \\
\hline MS 12 & TDZ & 1 \\
\hline MS 13 & TDZ & 1.5 \\
\hline MS 14 & TDZ & 2.0 \\
\hline MS 15 & TDZ & 2.5 \\
\hline
\end{tabular}

MS- Murashige and Skoog medium, BAP - Benzyl amino purine, KIN - Kinetin, TDZ -Thidiazuron

Table.2 Effect of type and concentration of cytokinin on shoot apical culture of Physalis minima L. (Mean \pm S.E.)

\begin{tabular}{|c|c|c|c|}
\hline Media & Establishment (\%) & $\begin{array}{c}\text { Elongation of existing shoot } \\
(\%)\end{array}$ & $\begin{array}{c}\text { Differentiation of multiple shoots } \\
(\%)\end{array}$ \\
\hline MS 1 & $86.11 \pm 0.56$ & $78.69 \pm 1.24$ & $62.54 \pm 0.53$ \\
\hline MS 2 & $\mathbf{9 6 . 1 1} \pm \mathbf{0 . 5 6}$ & $\mathbf{8 6 . 1 3} \pm \mathbf{0 . 9 4}$ & $\mathbf{7 5 . 1 5} \pm \mathbf{0 . 5 2}$ \\
\hline MS 3 & $83.33 \pm 0.96$ & $76.67 \pm 0.46$ & $65.34 \pm 0.38$ \\
\hline MS 4 & $91.11 \pm 0.56$ & $64.02 \pm 0.74$ & $60.98 \pm 0.53$ \\
\hline MS 5 & $87.22 \pm 0.55$ & $59.23 \pm 0.80$ & $43.94 \pm 0.87$ \\
\hline MS 6 & $81.67 \pm 0.96$ & $76.18 \pm 0.74$ & $70.06 \pm 0.77$ \\
\hline MS 7 & $83.89 \pm 0.56$ & $68.62 \pm 0.35$ & $65.36 \pm 0.38$ \\
\hline MS 8 & $95.56 \pm 0.56$ & $79.07 \pm 1.02$ & $69.76 \pm 0.69$ \\
\hline MS 9 & $78.33 \pm 0.96$ & $56.74 \pm 0.36$ & $47.52 \pm 0.36$ \\
\hline MS 10 & $\mathbf{7 5 . 0 0} \pm \mathbf{0 . 9 6}$ & $\mathbf{4 8 . 8 9} \pm \mathbf{1 . 1 1}$ & $\mathbf{4 1 . 4 8} \pm \mathbf{0 . 3 9}$ \\
\hline MS 11 & $83.89 \pm 2.42$ & $80.51 \pm 0.33$ & $70.15 \pm 0.99$ \\
\hline MS 12 & $82.78 \pm 0.554$ & $77.86 \pm 1.04$ & $68.45 \pm 0.80$ \\
\hline MS 13 & $91.67 \pm 0.96$ & $85.45 \pm 0.15$ & $72.720 \pm 0.29$ \\
\hline MS 14 & $88.33 \pm 0.96$ & $77.37 \pm 0.99$ & $71.69 \pm 0.31$ \\
\hline MS 15 & $78.89 \pm 1.11$ & $79.62 \pm 1.61$ & $64.07 \pm 0.51$ \\
\hline & C.D. 2.88 & C.D. 2.57 & C.D 1.72 \\
\hline & SE(m) 0.99 & SE(m) 0.88 & SE(m) 0.59 \\
\hline & SE(d) 1.41 & SE(d) 1.25 & SE(d) 0.84 \\
\hline & C.V. 2.01 & C.V. 2.10 & C.V. 1.62 \\
\hline
\end{tabular}

Each value represents mean of three replicates. Establishment was recorded 7 days after inoculation, elongation of existing shoot 10 days after elongation and formation of multiple shoot 20 days after inoculation. 
The elongation of existing shoot was significantly affected by the type and concentration of the cytokinin used in the medium. The medium supplemented with TDZ exhibited highest average frequency of shoot elongation (80.07) and lowest response was shown by medium with KIN (63.33). BAP at a concentration of $1.0 \mathrm{mg} / \mathrm{L}$ was the most effective cytokinin for elongation of existing shoot. The best concentration for this response for both TDZ and KIN was $1.5 \mathrm{mg} / \mathrm{L}$. These results are similar to earlier findings of Afroz et al., 2009; Intzaar et al., 2013; Solanki and Gupta, 2013; Sheeba et al., 2015 and Aniel Kumar et al., 2016 who found that the best response for shoot induction was obtained on the MS full strength basal medium supplemented with $1.0 \mathrm{mg} / \mathrm{L}$ BAP. However, Mungole et al., 2011 and Singh et $a l ., 2016$ observed that highest frequency of shoot induction was obtained on a medium having higher concentration of BAP $(2.5 \mathrm{mg} / \mathrm{L})$. The frequency of multiple shoot formation was less than shoot elongation but exhibited similar pattern. While TDZ was the most effective cytokinin for differentiation of multiple shoot, KIN was the least effective. MS 2 (BAP $1.0 \mathrm{mg} / \mathrm{L}$ ) was the best medium for differentiation of multiple shoot, MS 10 (KIN $2.5 \mathrm{mg} / \mathrm{L}$ ) was the least suitable. The most appropriate concentration for BAP was $1.0 \mathrm{mg} / \mathrm{L}$, for $\mathrm{KIN}$ was $0.5 \mathrm{mg} / \mathrm{L}$. TDZ was most effective at a concentration of $1.5 \mathrm{mg} / \mathrm{L}$ with highest frequency of differentiation of multiple shoot. The results are similar to the results of Afroz et al., 2009; Intzaar et al., 2013; Solanki and Gupta, 2013; Sheeba et al., 2015; Aniel Kumar et al., 2016 and Singh et al., 2016.

Our results indicated that at a very high or very low concentration, the responses of the shoot apical culture significantly decrease, indicating inhibitory effect of these concentrations. This result is supported by earlier findings of Aniel Kumar et al., 2016.
MS 2 was the best medium with highest frequency of all responses and MS 10 was the least effective medium with lowest frequency of all responses. While TDZ proved to be the best cytokinin with highest frequency of most of the responses, the response on medium with BAP was more intense. Thus, BAP at a concentration of $1.0 \mathrm{mg} / \mathrm{L}$ and TDZ at a concentration of $1.5 \mathrm{mg} / \mathrm{L}$ are equally effective for multiple shoot formation, hence micropropagation from shoot apical culture of $P$. minima. The multiple shoots developed directly from the shoot apices, without the formation of callus. Thus, the results of this investigation can be used to develop a very efficient micropropagation protocol for this economically important plant.

The effective induction and maintenance of multiple shoot from shoot apical culture of $P$. minima, depends on the type and concentration of cytokinin used in the medium. While TDZ was the most efficient cytokinin for development of multiple shoot in most of the cultures, BAP proved to be ideal for inducing more number of multiple shoots per explant. For each cytokinin, a concentration which was the most effective was identified. The results can be used to develop a highly efficient protocol for micropropagation of $P$. minima plants.

\section{Acknowledgement}

The authors wish to express their acknowledgement to Dr. Rajendra Prasad Central Agricultural University, Pusa, Samastipur, Bihar for providing the technical, human and financial support for conducting this work.

\section{Author Contributions}

All author have equally contributed to this article and its publication is approved by all authors. 


\section{References}

Afroz, F., Sayeed Hassan, A. K. M., Bari, L.S., Sultana, R., Begum, N., Akter Jahan, M.A. and Khatun, R. 2009.In vitro shoot proliferation and plant regeneration of Physalis minima L. - a perennial medicinal herb. Bangladesh Journal of Scientific and Industrial Research, 44: 453-456.

Aniel Kumar, O., Ramesh, S., Subba Tata, S. 2016. In Vitro Micropropagation of the Medicinal Plant Physalis angulata L. Not Sci Biol, 8(2):161-163. DOI:10.15835/nsb.8.2.98.

Anjani, K. and Kumar H. 2018. Morphological Features for Characterization of Local Populations of Physalis minima L. (Ban tipariya) in Bihar. International Journal of Agriculture Sciences, 10: 5047-5052.

Chothani, D. L., Vaghasiya, H. U. 2012. A phyto-pharmacological overview on Physalis minima Linn. Indian Journal of Natural Product and Resources, 3: 477482.

Intzaar, S., Akram, M. and Afrasiab, H.2013. High frequency multiple shoot formation of pygmy ground cherry (Physalis minima): An endangered medicinal plant. International Journal of Agriculture and Biotechnology, 15: 755-760.

Mungole, A. J., Doifode, V.D., Kamble, R. B., Chaturvedi, A. and Zanwar, P. 2011.
In-vitro callus induction and shoot regeneration in Physalis minima L. Annals of Biological Research,2: 79-85. Sheeba, E., Palanivel, S. and Parvathi, S. 2015.In vitro flowering and rapid propagation of Physalis minima Linn. A medicinal plant. International Journal of Innovative Research in Science, Engineering and Technology, 4: 18763 18768.

Singh, P., Singh, S. P., Shalitra, R., Samantaray, R., Singh, S. And Tiwary, A. 2016. In vitro regeneration of cape gooseberry (Physalis peruviana L.) through nodal segment. The Bioscan, 11(1): 41-44.

Solanki, A. and Gupta, D. 2013.In vitro shoot multiplication in Physalis minima var. Indica L. Biosciences Biotechnology Research Asia, 10: 371-374.

Ukwubile, C. A. and Oise, I. E. 2016 Analgesic and Anti-inflammatory Activity of Physalis angulata Linn. (Solanaceae) leaf methanolic extract in Swiss Albino mice. International Biology and Biomedical Journal, 2(4): 167-170.

Usaizan, N., Abdullah, N. A. P. and Saleh, G. 2014. Assessment of genetic diversity of Physalis minima L. (Solanaceae) based on ISSR marker. Journal of Applied Science and Agriculture, 9: 1825.

\section{How to cite this article:}

Kumari Anjani and Harsh Kumar. 2018. Effect of Cytokinin on Multiple Shoot Regeneration in Shoot Apical Culture of Physalis minima L. - An Important Fruit and Medicinal Plant. Int.J.Curr.Microbiol.App.Sci. 7(04): 3115-3121. doi: https://doi.org/10.20546/ijcmas.2018.704.353 